BICS 2004 Aug 29 - Sept 12004

\title{
Modelling the Interaction of Attention and Emotion
}

\author{
J. G. Taylor, N. Fragopanagos \\ Department of Mathematics, King's College \\ Strand, London WC2R 2LS, UK
}

\begin{abstract}
We review a recently developed engineering control approach to attention. The control system is extended to include biasing by emotional valence, with qualitative analysis given of a range of emotion paradigms. Application of the model is made to a recent paradigm underlining the need for attention in emotional influences. The paper ends with conclusion and discussion of further work.
\end{abstract}

\section{INTRODUCTION}

Attention is increasingly well studied in the human brain. It involves filtering out distracters from a desired target object or feature or spatial location by amplification of the target representations in low-level cortices, as well as by inhibition of distracter representations. The mechanism of such manipulation is currently under intensive study. At the same time the interaction of attentive and emotion-based processing is also being studied, with some level of inhibition between the two being observed. These features impact on our understanding of the twin pillars of human information processing ('thinking' and 'feeling') as well as on attempts to incorporate emotions into artificial agents. They are also relevant to guide the development of artificial systems recognising emotions in humans (as being pursued by ERMIS [1]). A control model of attention will be reviewed in this paper, and extended to the addition of valence as an emotional component. This will then be applied to a specific divided attention paradigm [31], to explain the effects of attention on activation of emotional valence in the amygdala. This is extended to other modules, as well as detailed temporal predictions being made in this paradigm.

\section{ATTENTION}

Numerous experiments in brain imaging have demonstrated that there is a network of cortical modules involved in goaldirected control of attention [2,3]. There is some overlap of the network for attention controlled by external rapidly appearing inputs (so-called exogenous attention) and the topdown or endogenous form controlled by internal goals $[4,5$, 6]. The most important cortical components of this attention network have been proposed as being the parietal lobes (for perceptual processing), the anterior cingulate (for limbicbased intention), and the prefrontal cortex (for rules and templates for guiding response) [7]. A recent review [8] concluded that "Attention-related activity in frontal and parietal areas does not reflect attention modulation of visually evoked response, rather it reflects the attention operations themselves." More recently ([9] p 201), in their wide-ranging review of attention, concluded: "One system, which includes part of the intra-parietal cortex and superior frontal cortex, is involved in preparing and applying goal-directed (top-down) selection for stimuli and responses." However they point out that there is another, partly overlapping, network crucially involved in exogenous attention, including ventral components: the tempero-parietal junction and ventral frontal cortex, both in the right hemisphere, acting as a 'circuit breaker' for detecting attention to salient events. We will turn to this later.

We may summarize the overall control feature of attention by a two-network model:

\section{CONTROLLING NETWORK OF MODULES $\downarrow$}

CONTROLLED NETWORK OF MODULES

The down-most modules in (1) are the regions of the brain which are controlled by attention, consisting of lower regions - especially in occipital, temporal and motor cortex - in which input or response activities are modulated by attention, but these areas do not function so as to control attention in any strong manner (barring areas, such as the amygdala, which code for input valence, so affecting where attention is directed). The other set of modules, termed 'controller' in (1); create the attention control signal itself. They are composed of 'higher' areas, especially the prefrontal and parietal areas of cortex.

Attention has been modelled in a variety of ways: by regarding it as a filter [10] or as a mechanism to bind together different pre-attentive components of objects [11]. More detailed models of its mechanics have been in terms of 'biased competition' on lower cortical sites, the bias arising from top-down goal sites [12], and by more detailed neural network models of such biasing [13, 14, 15]. All these models can be subsumed under a more general engineering control approach, as developed in $[16,17,18,19]$, as will now be reviewed.

Given that attention functions by signals generated by a separate control region sent to modulate the neural activity in controlled regions, as in (1), the creation of such modulation itself will involve internal complexity. The variety of distinct functional components in engineering control models (goal sites, forward models or observers, inverse model controllers, error monitors) leads one to expect a similar range of functions being performed by the component brain areas observed in attention. The network of cortical modules for overall attention control (prefrontal, parietal), as well as the cortical recipient sites of the control signals needed to achieve 
the filtering process basic to attention from the higher-level sites, are ripe for analysis by engineering control concepts.

Effective control proceeds by creating suitable control signals to influence the 'plant' (being controlled) so that it develops in time through a desired set of states. We can identify, for attention, the plant as the lower level cortices on which there are representations of the environment. The control signal is to be generated so that a particular target activity in lower cortex is amplified, other activity reduced (although not annihilated). Control signal generation, state estimation, and feedback of errors from the plant to correct the control signal are the main tools to achieve this desired control. Control uses state estimators, feedback assessors, error monitors, control action generators and goal storage modules. We can as sume that the brain, under evolutionary pressure, has used the notions in developing suitable modules with these functions. There is good evidence, from many experimental analyses, of the existence of these modules with the suggested functionality. Figure 1 shows a simplified control model along these lines for sensory-motor control.

A particular feature of relevance in figure 1 is the presence of the IMC as separate from the goal map and from the underlying plant (low-level cortical) module. There is strong evidence for this separation from [9], with sites in Pre-Frontal Cortex (PFC) as well as parietal lobe being involved in cued attention movement. Since the PFC has goal-like properties, we can safely assume that the parietal sites involved IMC-like activity.

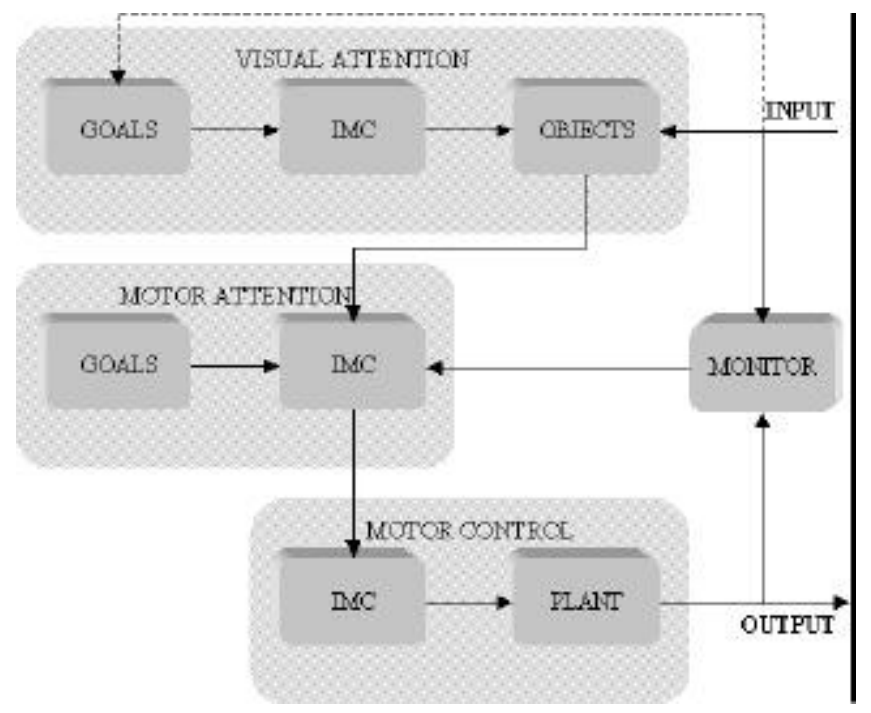

Fig. 1. The Sensory-Motor Attention control model: IMC $=$ inverse model controller

\section{EMOTION \& ATTENTION}

Emotional experience is known to have two components: 'automatic' and 'attended', similar to the division of attention. Automatic emotions activate paralimbic regions (amygdala, hypothalamus), so more medial in the brain, and may remain outside awareness. Attended emotion, on the other hand, activates heteromodal cortical sites involved in aware processing (parietal, prefrontal, cingulate). Thus we can preliminarily divide emotion into a valence component (in paralimbic) and an attention-grabbing component, leading to emotional awareness, or feeling. This latter could be driven by the nucleus basalis Meynert (NBM), a source of Ach; however NBM has a large input from sites coding for reward values of inputs, so involving valence driving attention.

Brain imaging/deficit results in depressives indicate the division of processing into: a ventral network (for emotion) and a dorsal one (for cognition), where imbalance between the two leads to reduction of the cognitive activity and an excess of limbic activity. This leads to the question as to the nature of the interaction between emotion and attention: are they competing 'attention-type' systems, or is attention the main control system, but guided by emotional valence? To answer that we will develop an architecture for their interaction by extending the sensory-motor attention control model of figure 1 and the discussion of the previous section.

We start with the amygdala, a particularly important site for fast valuation of stimuli. It is able to achieve that by being reciprocally well coupled to posterior as well as anterior cortical sites. Thus the amygdala can bias attention by added activation, in particular for stimuli with negative valence. A further important component is the orbito-frontal cortex (OFC), which has been observed in interaction with dorsal PFC sites of endogenous attention. In particular a paradigm involving comparison of the activities of these sites when erotic videos were viewed led to reciprocal inhibition between these two regions (DLPFC versus OFC) [27]. Similar mutual inhibition was observed in related paradigms [28, 29, 30]. In these studies activations of the ventral PFC were also reported as related to enhanced OFC activity, so implicating the right ventral attention orienting circuit mentioned earlier as coupled to the valence circuit. We also take support for the architecture of figure 1 from the model of Mayberg [34], especially the inclusion of an inhibitory interaction between these two components (OFC \& DLPFC).

The overall attention/emotion network can thus be constructed as shown in figure 2 .

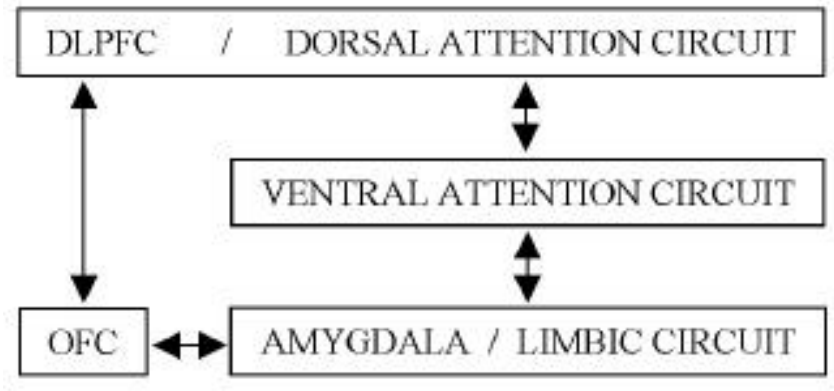

Fig. 2. The overall attention/emotion network 
We have delineated in figure 2 the two attention circuits discussed in section 2, as well as included the amygdala and OFC. Further modules (hypothalamus, striatal components) are also involved but their role is beyond this paper. The question raised earlier can be rephrased in terms of the diagram of figure 2 as: Does the emotion-creating limbic circuit of amygdale and OFC have an independent control function of similar character to the ventral and dorsal attention circuits? A related question, still controversial, is as to the need for attention to an emotionally-laden stimulus to cause amygdala activation; presently it appears as if that is the case [31]. In other words, expression of the valence of a stimulus requires attention to it.

Let us look at the overall connectivity between the three circuits (dorsal and ventral attention and limbic circuits), using the results from these various experiments, to explore the architecture of figure 2 further:

1) AMYG is excitatorily reciprocally connected to CX, so to both ventral and dorsal attention circuits. It has fast activation from posterior sites in stimulus processing, which may allow it's early sending of a valence tag to these sites, and to the ventral or dorsal sites of attention control IMCs in parietal lobes [9]. There is also evidence on amygdala-based boosting of attention resources, as detected in the emotional attentional blink [32]; a simulation of this effect is presented later in the session [33];

2) OFC is excitatorily coupled to the PFC component of the ventral attention circuit, but inhibitorily coupled to the dorsal partner [29]...

With these additional features, together with results on depression from [34] we conclude that the bottomlevel emotion circuit of figure 2 normally functions as an additional valence tagging system for the ventral circuit, so leading to enhanced circuit-breaking of the dorsal attention control system. It achieves this, I propose, not only by activating the ventral components (especially the goal sites in ventral PFC) but it also reduces, by inhibition, cognitive goals present in DLPFC. In depression, cognitive goals are unable to be effective in normal situations, due to continued inhibition from the OFC hyper-activity.

\section{SIMULATING THE INTERACTION OF ATTENTION AND EMOTIONS}

We consider the paradigm of [31]. This used fMRI to measure whole brain activity when subjects viewed pictures of fearful, neutral or happy faces under a difficult divided attention to either the face or by comparing the orientation of peripherally-placed bars (the faces were presented in the centre of vision, which was fixated by the subjects). Amygdala was only observed active under attention to the emotionally-charged faces (with stronger effects in right amygdale from fearful than happy faces). The face ventral temporal lobe area was also activated by attention, again more strongly by fearful faces, as also occurred in the OFC/ventral PFC. They noted effects of modulation by amygdala on a number of these cortical sites: ventral PFC.OFC, and early visual cortex. The architecture of figure 2 explains these modulation effects qualitatively, provided there is a stronger coding of fearful face in the right AMYG. would then be in the limbic component of figure 2 , in addition to feedback effects from physiological changes in the body and over cortical regions from amygdala modulation [35].

We now turn to a more detailed quantitative analysis of the paradigm of [31]. This uses two sets of 'objects' for detailed analysis and recognition: one is oriented bars, the other (on a separate map) is of faces. Only the latter set has emotional valence attached to it. Thus only are there excitatory connections between the amygdala and

\section{EMOTIONAL RECOGNITION}

The architecture of figure 2, proposed here as at the basis of human emotion experience, is to be expected also to be crucially involved in the recognition of emotion in others, and especially of the basic emotions (fear, anger, disgut, sadness, happiness and surprise), as supported by the various paradigms described above, in which the network of figure 2 was activated in various ways when pictures of emotionally charged faces were observed by subjects. There are further details that have to be considered in analysing the architectural differences between recognising emotions in faces or speech, such as the complimentarity between brain sites for recognition through prosody or linguistic content [36] (and the difficulty of using prosody in comparison to facial features), or the two different mechanisms of emotion recognition discussed in [37], of construction of a simulation of the emotion in the perceiver, or the modulation of sensory cortices by top-down influences.

However these aspects do not change the basic structure of the architecture of figure 2 as common to basic emotional experience and analysis. We have used the architecture of figure 2 to develop a neural network for emotion recognition which includes explicit feedback to the input layer to modulate those features important for the experience of a specific emotion [36]. The possibility of using all three forms of input (facial, linguistic and prosodic) is at the basis of ERMIS, as described in later talks.

\section{CONCLUSIONS}

We have reviewed a control approach to attention which allows the detailed assignment of various control functions to separate components of the network of sites observed in the brain during attention paradigms. Emotions were then folded into the attention control model by adding amygdala and orbito-frontal components. The interactions of these sites with the separate ventral (exogenous) and dorsal (endogenous) attention networks were considered, and used to analyse an experimental paradigm in which the interaction of emotion and attention was very clearly delineated. The paradigm was simulated, with results agreeing with those of experiment, but 
going beyond them in other regions and in temporal sensitivity. We finally and briefly considered the relevance of these results to emotion recognition. The overall conclusion is that it is not possible to consider emotion processing without inclusion of attention, and conversely that emotion functions importantly to help guide attention.

\section{ACKNOWLEDGEMENTS}

This word has been partially supported by the European Commission under the ERMIS Project Grant (IST-200029319) and the ORESTEIA Project Grant (IST-2000-26091).

[1] http://www.image.ntua.gr/ermi

\section{REFERENCES}

[2] Hopfinger, J. B., Buonocore, M. H., and Mangun, G. R., "The neural mechanisms of top-down attentional control," Nat.Neurosci., vol. 3, no. 3, pp. 284-291, Mar.2000.

[3] Hopfinger, J. B., Woldorff, M. G., Fletcher, E. M., and Mangun, G. R. "Dissociating top -down attentional control from selective perception and action," Neuropsychologia, vol. 39, no. 12, pp. 1277-1291, 2001.

[4] Gitelman, D. R., Nobre, A. C., Parrish, T. B., LaBar, K. S., Kim, Y. H., Meyer, J. R., and Mesulam, M., "A large-scale distributed network for covert spatial attention: further anatomical delineation based on stringent behavioural and cognitive controls," Brain, vol. 122 ( Pt 6) pp. 1093-1106, June1999.

[5] Kim, Y. H., Gitelman, D. R., Nobre, A. C., Parrish, T. B., LaBar, K. S., and Mesulam, M. M., "The large-scale neural network for spatial attention displays multifunctional overlap but differential asymmetry," Neuroimage., vol. 9, no. 3, pp. 269-277, Mar.1999.

[6] Coull, J. T. and Nobre, A. C., "Where and when to pay attention: the neural systems for directing attention to spatial locations and to time intervals as revealed by both PET and fMRI," J.Neurosci., vol. 18, no. 18, pp. 74267435, Sept.1998.

[7] Mesulam, M. M., "A cortical network for directed attention and unilateral neglect," Ann.Neurol., vol. 10, no. 4, pp. 309-325, Oct.1981.

[8] Kastner, S. and Ungerleider, L. G., "The neural basis of biased competition in human visual cortex," Neuropsychologia, vol. 39, no. 12, pp. 1263-1276, 2001

[9] Corbetta, M. and Shulman, G. L., "Control of goal-directed and stimulusdriven attention in the brain," Nat.Rev.Neurosci., vol. 3, no. 3, pp. 201-215, Mar.2002.

[10] Broadbent, D. E, Perception and communication London: Pergamon Press, 1958

[11] Treisman, A. M., "Strategies and models of selective attention," Psychol.Rev., vol. 76, no. 3, pp. 282-299, May1969.

[12] Desimone, R. and Duncan, J., "Neural mechanisms of selective visual attention," Annu.Rev.Neurosci., vol. 18 pp. 193 -222, 1995.

[13] Deco, G., "Biased Competition Mechanisms for Visual Attention," in Wermter, S., Austin, J., and Willshaw, D. (eds.) Emergent Neural Computational Architectures Based on Neuroscience - Towards Neuroscience-Inspired Computing Heidelberg: Springer, 2001, pp. 114-126.

[14] van der Velde, F. and de Kamps, M., "From knowing what to knowing where: modeling object-based attention with feedback disinhibition of activation," J.Cogn Neurosci., vol. 13, n o. 4, pp. 479-491, May2001.

[15] Mozer, M. C. and Sitton, M., "Computational modeling of spatial attention," in Pashler, H. (ed.) Attention New York: Taylor \& Francis, 1999, pp. 341-393.

[16] Taylor, J. G., "Attentional movement: the control basis for consciousness," Society for Neuroscience Abstracts [26], 2231\#839.3, 2000.

[17] Taylor, J. G., "From Matter to Mind," Journal of Consciousness Studies, vol. 9, no. 4, pp. 3-22, 2002.

[18] Taylor, J. G. and Rogers, M., "A control model of the movement of attention," Neural Netw., vol. 15, no. 3, pp. 309-326, Apr.2002.

[19] Taylor, J. G., Progress in Neurobiology, vol. 71, no. 4, 2003.
[27] Beauregard, M., Levesque, J., and Bourgouin, P., "Neural correlates of conscious self-regulation of emotion," J.Neurosci., vol. 21 , no. 18, pp. RC165, Sept.2001.

[28] Armony, J. L. and Dolan, R. J., "Modulation of spatial attention by fear-conditioned stimuli: an event-related fMRI study," Neuropsychologia, vol. 40, no. 7, pp. 817-826, 2002.

[29] Yamasaki, H., LaBar, K. S., and McCarthy, G., "Dissociable prefrontal brain systems for attention and emotion," Proc.Natl.Acad.Sci.U.S.A, vol. 99, no. 17, pp. 11447-11451, Aug.2002.

[30] Perlstein, W. M., Elbert, T., and Stenger, V. A., "Dissociation in human prefrontal cortex of affect ive influences on working memory-related activity," Proc.Natl.Acad.Sci.U.S.A, vol. 99, no. 3, pp. 1736-1741, Feb. 2002.

[31] Pessoa, L., McKenna, M., Gutierrez, E., and Ungerleider, L. G., "Neural processing of emotional faces requires attention," Proc.NatlAcad.Sci.U.S.A, vol. 99, no. 17, pp. 11458-11463, Aug.2002.

[32] Anderson, A. K. and Phelps, E. A., "Lesions of the human amygdala impair enhanced perception of emotionally salient events," Nature, vol. 411, no. 6835, pp. 305-309, May2001.

[33] Fragopanagos, N. and Taylor, J. G., "The Effect of Emotional Bias in Attentional Processes," Proceedings of IJCNN 2004 (to be published), 2004.

[34] Mayberg, H. S., "Limbic-cortical dysregulation: a proposed model of depression," J.Neuropsychiatry Clin.Neurosci., vol. 9, no. 3, pp. 471-481, 1997.

[35] Taylor, J. G., Fragopanagos, N., Cowie, R., Douglas-Cowie, E., Fotinea, S. -E., and Kollias, S., "An Emotional Recognition Architecture based on Human Brain Structure," Proceedings of the 13th International Conference on Artificial Neural Networks \& 10th International Conference on Neural Information Processing, pp. 1133-1140, 2003.

[36] McNeely, H. E. and Parlow, S. E., "Complementarity of linguistic and prosodic processes in the intact brain," Brain Lang, vol. 79, no. 3, pp. 473 481, Dec.2001.

[37] Adolphs, R. "Neural systems for recognizing emotion," Curr.Opin.Neurobiol., vol. 12, no. 2, pp. 169-177, Apr.2002. 